01

\title{
Особенности моделирования возмущений магнитного поля электрофизических установок стальной арматурой зданий
}

\author{
(C) В.М. Амосков, ${ }_{1}^{1}$ А.М. Базаров, ${ }^{1}$ В.А. Беляков, ${ }^{1,2}$ Е.И. Гапионок, ${ }^{1}$ М.В. Капаркова, ${ }^{1}$ В.П. Кухтин, ${ }^{1}$ \\ Е.А. Ламзин, ${ }^{1}$ Б.В. Люблин, ${ }^{1}$ Д.А. Овсянников, ${ }^{2}$ C.Е. Сычевский ${ }^{1,2}$ \\ ${ }^{1}$ АО НИИ электрофизической аппаратуры им. Д.В. Ефремова, \\ 196641 Металлострой, Санкт-Петербург, Россия \\ ${ }^{2}$ Санкт-Петербургский государственный университет, \\ 199034 Санкт-Петербург, Россия \\ e-mail: sytch@sintez.niiefa.spb.su
}

(Поступило в Редакцию 5 декабря 2016 г. В окончательной редакции 15 апреля 2017 г.)

Предложен эффективный способ моделирования стальной арматуры в корпусах зданий электрофизических установок с целью точного учета возмущения магнитного поля, вызванного намагничиванием стержней. Решетка из арматуры представлена одним или несколькими слоями однородного изотропногоматериала с предварительно вычисленными эквивалентными (усредненными) магнитными свойствами. Приведены примеры расчета таких магнитных свойств как с использованием упрощенного аналитического подхода, так и с помощью численного моделирования магнитного поля в трехмерной ячейке периодичности решетки арматуры. Эффективность способа продемонстрирована на важном практическом примере моделирования возмущения однородного магнитного поля армированной плитой. Выполнено сравнение с результатами моделирования, использующими другие подходы.

DOI: 10.21883/JTF.2017.10.44987.2124

\section{Введение}

Бетонные конструкции зданий электрофизических установок содержат стальную арматуру и массивные стальные элементы. Например, здания токамака, входящие в состав Комплекса токамака ИТЭР, содержат стальную арматуру с периодической пространственной структурой (рис. 1 [1]). Процентное содержание стали в таких конструкциях варьирует от 1.5 до $12 \%$ [2]. Внутренние элементы здания токамака частично располагаются на сравнительно небольших (5-10 m) расстояниях от токамака. Стальные стержни арматуры могут намагничиваться полями рассеяния электромагнитной системы ИТЭР и плазмы, вплоть до насыщения.

Ранее проведенные расчеты показали [3] заметное влияние намагничивания арматуры на распределение магнитного поля внутри камеры установки ИТЭР в области плазмы, которая чувствительна к возмущениям полей малого уровня.

Актуальным является вопрос электромагнитной совместимости оборудования в рабочих помещениях здания ИТЭР [4].

В подобных расчетах использовались глобальные вычислительные модели, которые включали как элементы конструкции, изготовленные полностью из стали, так и бетонные элементы, армированные стальными стержнями.

Авторами настоящей работы разработана глобальная вычислительная модель здания токамака ИТЭР MMTC1 (Magnetic Model of Tokamak Complex, ver. 1) [2].

Одной из проблем при построении детальной расчетной модели здания токамака является невозможность учета расположения отдельных стержней арматуры, создающих сложную картину распределения поля внутри армированных элементов здания или вблизи них. При этом уже на расстояниях, сравнимых с характерным шагом укладки стержней, влияние каждого отдельного стержня становится малоразличимым. Конструкция с арматурой в таком случае может быть представлена как непрерывный континуум с усредненными магнитными свойствами. Эти свойства определяются не только магнитными свойствами арматуры, но и особенностями укладки стержней.

В ММТС1 все элементы здания заменены эквивалентным однородным изотропным материалом, свойства которого описывались зависимостью магнитной индукции $B=0.5 k f(H)$ от напряженности поля $H$, где $B=f(H)$ - кривая намагничивания арматуры, а $k-$

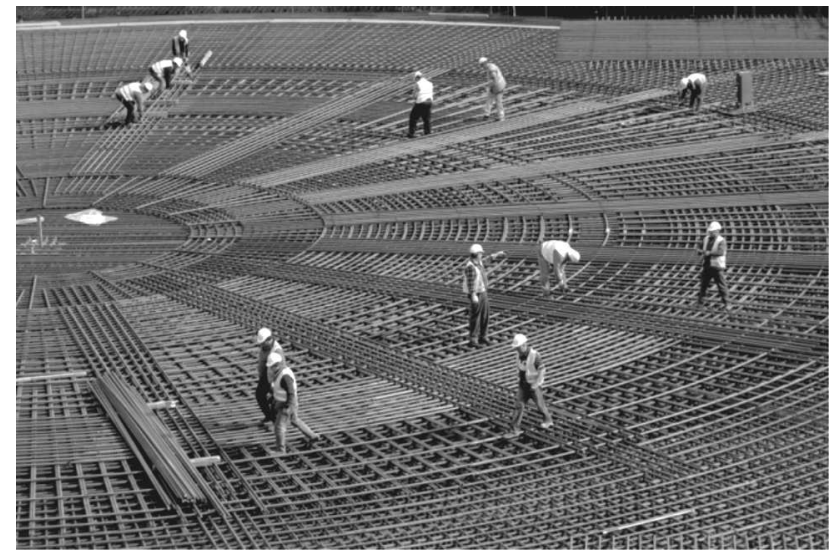

Рис. 1. Положение слоев арматуры крест-накрест [1]. 
объемный коэффициент заполнения бетона арматурой. Коэффициент $\eta=0.5$ учитывает особенности типичной укладки стержней в двух взаимно-перпендикулярных направлениях, определяющих плоскость укладки, в которой только $50 \%$ стержней арматуры могут эффективно проводить магнитный поток во внешнем поле, ориентированном вдоль стержней. Можно показать, что величина $\eta$ принимает значение 0.5 в случае, когда поле параллельно плоскости укладки арматуры. Это утверждение перестает быть справедливым, если поле имеет компоненту, перпендикулярную плоскости укладки арматуры. В этом направлении отсутствуют стержни, эффективно проводящие магнитный поток, и как следствие, намагничение материала в соответствующем направлении должно быть слабым. На основании анализа распределения поля в модели ММТС1 можно предположить, что влияние этой компоненты проявляется сравнительно слабо. Тем не менее модель ММТС1 требует более надежного обоснования и при необходимости уточнения.

В настоящей работе предложен более точный метод учета влияния арматуры, позволяющий оставаться в рамках однородной изотропной модели.

Приведены результаты численного решения задачи о возмущении однородного внешнего поля периодической структурой, образованной стальными стержнями, с использованием трех моделей:

1 - детальной, в которой учитываются форма, положение и свойства каждого стержня;

2 - изотропной, в которой решетка стержней заменена эквивалентным однородным изотропным материалом, как при разработке МMTC1;

3 - модифицированной изотропной, соответствующей предложенному подходу.

Расчеты 3D распределений магнитного поля выполнены комплексом программ КОМРОТ [5,6], использующего метод конечных элементов. Верификация модели выполнена путем сопоставления с расчетами комплексом программ KLONDIKE [7], базирующимся на интегральном подходе.

\section{Анизотропная модель решетчатой структуры}

Типичным является расположение арматуры слоями, в каждом из которых стальные стержни уложены практически параллельно друг другу с одинаковым шагом. Направления укладки в соседних слоях взаимно перпендикулярны. Расстояния между слоями - постоянная величина. Минимальной ячейкой периодичности этой структуры является гексаэдр со сторонами, равными шагу укладки арматуры.

В типичном случае размер ячейки существенно меньше характерных размеров армированных конструкций, и с уменьшением относительных размеров ячеек распределения поля в близлежащих ячейках все менее отличаются друг от друга. В пределе распределение поля внутри ячейки определяется: 1 - ее структурой, 2 - вектором ее среднего поля и 3 - граничными условиями периодичности. В этом случае можно перейти от использования ячеистой структуры к эквивалентной гомогенной среде. Напряженность и индукция в точках эквивалентной гомогенной среды определяются средними значениями напряженностей и индукций внутри ячеек. Анизотропные свойства эквивалентной среды определяются отношением средней индукции к средней напряженности в ячейке при различных заданных направлениях среднего поля.

Таким образом, исходная задача детального определения поля в ячеистой (решетчатой) структуре может быть упрощена и разделена на два этапа: первый - определение анизотропных свойств эквивалентных сред для каждого способа укладки арматуры путем нахождения распределений поля в ячейках, и, после этого, нахождение поля в глобальной модели с заменой решетчатых структур на эквивалентные анизотропные однородные среды - второй.

В приближении постоянных магнитных свойств задача расчета поля является линейной. Поле в ячейке может быть представлено в виде суперпозиции решений трех задач для каждой из компонент среднего поля. Эти компоненты определены в локальной системе прямоугольных декартовых координат $X Y Z$, оси которой параллельны ребрам ячейки. Задавшись в общем случае направлением и величиной напряженности среднего поля $H$ в ячейке (с использованием соответствующих граничных условий) для каждой задачи можно определить среднюю индукцию $B$. Средняя (эффективная) магнитная проницаемость ячейки в каждом направлении определяется отношением средних величин индукции и напряженности.

Очевидно, что в линейном случае это отношение зависит не от уровня поля, но от его направления. Магнитные проницаемости в характерных направлениях определяют анизотропные свойства однородной среды, которые описываются постоянным симметричным тензором с отличными от нуля диагональными компонентами $-\mu_{x x}, \mu_{y y}, \mu_{z z}$.

В случае нелинейных свойств арматуры можно принять, что в ячейке периодичности магнитная проницаемость в любой точке стержней одинакова. При этом ее величина определяется средней напряженностью поля ячейки согласно кривой намагниченности $\mu(H)$ материала стержней. В этом случае для любой наперед заданной величины проницаемости $\mu$, задача становится линейной. Таким образом, нелинейные анизотропные свойства эквивалентной среды могут быть описаны функциейтензором, которая зависит только от магнитной проницаемости стержней: $\left(\mu_{x x}, \mu_{y y}, \mu_{z z}\right)=f(\mu)=f(\mu(H))$.

Поскольку структура ячейки может быть произвольной, то ее эффективные свойства должны определяться для конкретного случая путем моделирования поля в ячейке. 

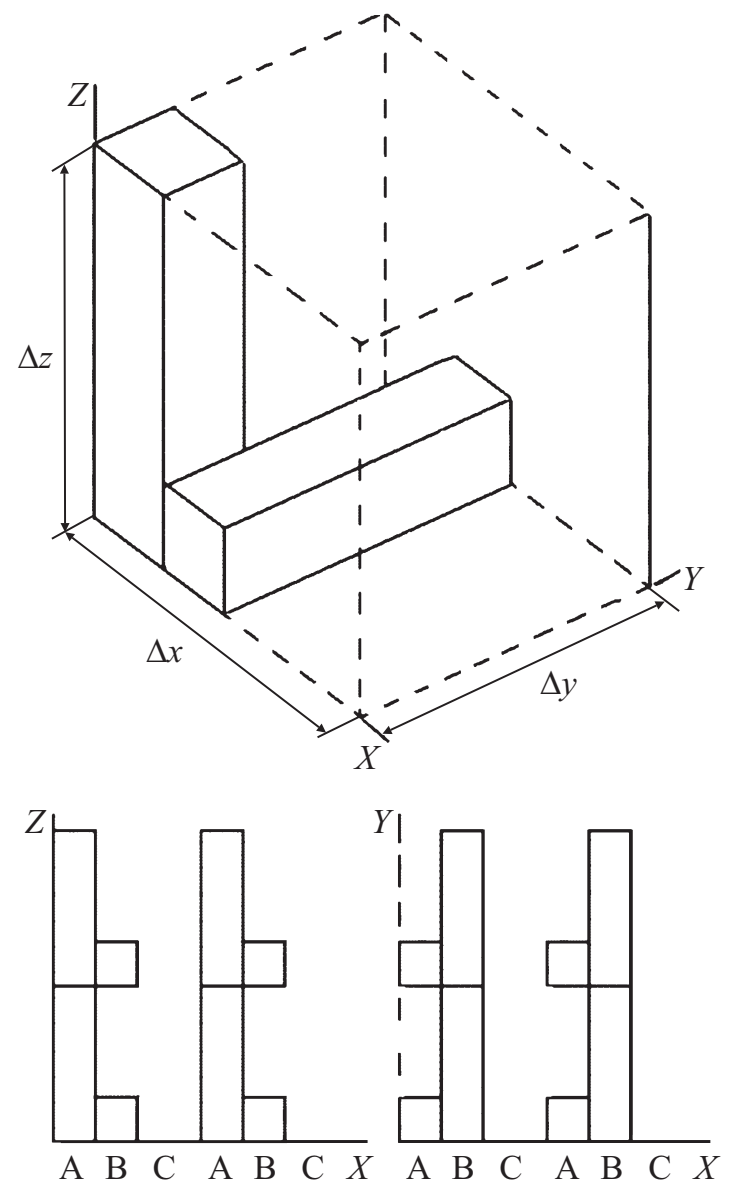

Рис. 2. Слои в периодической структуре арматуры для вывода упрощенных соотношений.

Введем систему декартовых координат $X, Y, Z$. Будем считать, что слои стержней параллельны плоскости $Y Z$ и в половине из них (в нечетных слоях) стержни уложены вдоль оси $Z$ с шагом $\Delta y$, а в другой половине (в четных слоях) - вдоль оси $Y$ с шагом $\Delta z$. Шаг укладки пар слоев $\Delta x$. Примем для удобства на начальном этапе, что стержни в сечении представляют собой прямоугольник.

Ячейка периодичности представляет собой прямоугольный параллелепипед с размерами $\Delta x \times \Delta y \times \Delta z$, состоящий в направлении оси $X$ из трех слоев (рис. 2): слой А, толщиной $\Delta x_{\mathrm{A}}$, в котором стержни с сечением $\Delta x_{\mathrm{A}} \times \Delta y_{\mathrm{A} 1}$ параллельны оси $Z$, расстояние между стержнями в слое $\Delta y_{\mathrm{A} 0}=\Delta y-\Delta y_{\mathrm{A} 1}$; слой $\mathrm{B}$, толщиной $\Delta x_{\mathrm{B}}$, в котором стержни с сечением $\Delta x_{\mathrm{B}} \times \Delta z_{\mathrm{B} 1}$ параллельны оси $Y$, расстояние между стержнями в слое $\Delta z_{\mathrm{B} 0}=\Delta z-\Delta z_{\mathrm{B} 1}$; слой С толщиной $\Delta x_{\mathrm{C}}=\Delta x-\Delta x_{\mathrm{A}}-\Delta x_{\mathrm{B}}$, без стержней.

Введем $B_{x}, B_{y}, B_{z}, H_{x}, H_{y}, H_{z}, \mu_{x}, \mu_{y}, \mu_{z}$, соответствующие эффективным (средним) величинам рассматриваемого трехслойного элемента с анизотропией магнитных свойств. Введем $B_{j \mathrm{~A}}, B_{j \mathrm{~B}}, B_{j \mathrm{C}}, H_{j \mathrm{~A}}, H_{j \mathrm{~B}}, H_{j \mathrm{C}}$, $\mu_{j \mathrm{~A}}, \mu_{j \mathrm{~B}}, \mu_{j \mathrm{C}}$ (где $j=x, y$, или $z$ ), соответствующие эффективным величинам для каждого из слоев. Через
$B_{j \mathrm{~A} 1}, B_{j \mathrm{~B} 1}, H_{j \mathrm{~A} 1}, H_{j \mathrm{~B} 1}, \mu_{\mathrm{A} 1}, \mu_{\mathrm{B} 1}$ обозначим соответствующие величины в стержнях, а через $B_{j \mathrm{~A} 0}, B_{j \mathrm{~B} 0}, B_{j \mathrm{C} 0}$, $H_{j \mathrm{~A} 0}, H_{j \mathrm{~B} 0}, H_{j \mathrm{C} 0}, \mu_{\mathrm{A} 0}=\mu_{\mathrm{B} 0}=\mu_{\mathrm{C}}=1-$ в вакууме в соответствующем слое.

Рассмотрим в качестве примера Z-компоненту, слой А:

$$
B_{z \mathrm{~A} 1}=\mu_{0} \mu_{\mathrm{A} 1} H_{z \mathrm{~A} 1}, B_{z \mathrm{~A} 0}=\mu_{0} H_{z \mathrm{~A} 0}, B_{z \mathrm{~A}}=\mu_{0} \mu_{z \mathrm{~A}} H_{z \mathrm{~A}} .
$$

В этом слое Z-компонента является касательной к границе раздела сред. Поэтому

$$
H_{z \mathrm{~A} 1}=H_{z \mathrm{~A} 0}=H_{z \mathrm{~A}} .
$$

Среднее значение индукции $B_{z \mathrm{~A}}$ в сечении $S_{z \mathrm{~A}}=$ $=\Delta_{x \mathrm{~A}} \Delta y$ определяется через поток $\Phi_{z \mathrm{~A}}$ в этом сечении: $B_{z \mathrm{~A}}=\Phi_{z \mathrm{~A}} / S_{z \mathrm{~A}}$. Поток равен

$$
\Phi_{z \mathrm{~A}}=\Phi_{z \mathrm{~A} 1}+\Phi_{z \mathrm{~A} 0}=B_{z \mathrm{~A} 1} S_{z \mathrm{~A} 1}+B_{z \mathrm{~A} 0} S_{z \mathrm{~A} 0},
$$

где $S_{z \mathrm{~A} 1}=\Delta x_{\mathrm{A}} \Delta y_{\mathrm{A} 1} \quad$ и $S_{z \mathrm{~A} 0}=\Delta x_{\mathrm{A}} \Delta y_{\mathrm{A} 0}$. Введем для слоя А коэффициент заполнения $k_{y \mathrm{~A}}=\Delta y_{\mathrm{A} 1} / \Delta y$. Тогда

$$
\begin{aligned}
B_{z \mathrm{~A}} & =\left(\Delta y_{\mathrm{A} 1} B_{z \mathrm{~A} 1}+\Delta y_{\mathrm{A} 0} B_{z \mathrm{~A} 0}\right) / \Delta y \\
& =k_{y \mathrm{~A}} B_{z \mathrm{~A} 1}+\left(1-k_{y \mathrm{~A}}\right) B_{z \mathrm{~A} 0} .
\end{aligned}
$$

Разделив (3) на $\mu_{0} H_{z \mathrm{~A}}$ и используя соотношения (1) и (2), получим

$$
\mu_{z \mathrm{~A}}=k_{y \mathrm{~A}} \mu_{\mathrm{A} 1}+\left(1-k_{y \mathrm{~A}}\right)=1+k_{y \mathrm{~A}}\left(\mu_{\mathrm{A} 1}-1\right) .
$$

Выполнив соответствующие выкладки для слоев В, С и использовав обозначения $k_{z \mathrm{~B}}=\Delta z_{\mathrm{B} 1} / \Delta z, k_{x \mathrm{~A}}=$ $=\Delta x_{\mathrm{A}} / \Delta x$ и $k_{x \mathrm{~B}}=\Delta x_{\mathrm{B}} / \Delta x$, можно получить

$$
\begin{aligned}
\mu_{z}= & 1+k_{x \mathrm{~A}} k_{y \mathrm{~A}}\left(\mu_{\mathrm{A} 1}-1\right) \\
& +k_{x \mathrm{~B}}\left(1 /\left(1-k_{z} \mathrm{~B}\left(1-1 / \mu_{\mathrm{B} 1}\right)\right)-1\right),
\end{aligned}
$$

Аналогичные рассуждения приводят к выражениям для $Y$ - и $x$-компонент:

$$
\begin{aligned}
\mu_{y}=1 & +k_{x \mathrm{~A}}\left(1 /\left(1-k_{y \mathrm{~A}}\left(1-1 / \mu_{\mathrm{A} 1}\right)\right)-1\right) \\
+ & k_{x \mathrm{~B}} k_{z \mathrm{~B}}\left(\mu_{\mathrm{B} 1}-1\right), \\
1 / \mu_{x}= & 1-k_{x \mathrm{~A}}\left(1-1 /\left(1+k_{y \mathrm{~A}}\left(\mu_{\mathrm{A} 1}-1\right)\right)\right) \\
& -k_{x \mathrm{~B}}\left(1-1 /\left(1+k_{z} \mathrm{~B}\left(\mu_{\mathrm{B} 1}-1\right)\right)\right) .
\end{aligned}
$$

Рассмотрим частный случай $\mu_{\mathrm{A} 1}=\mu_{\mathrm{B} 1}=\mu_{1}$. Введем фактический коэффициент заполнения сталью: $k=$ $=k_{x \mathrm{~A}} k_{y \mathrm{~A}}+k_{x \mathrm{~B}} k_{z}$ и и эффективные коэффициенты заполнения по каждому из трех направлений:

$$
\begin{gathered}
k_{z}=\left(\mu_{z}-1\right) /\left(\mu_{1}-1\right), \quad k_{y}=\left(\mu_{y}-1\right) /\left(\mu_{1}-1\right), \\
k_{x}=\left(\mu_{x}-1\right) /\left(\mu_{1}-1\right) .
\end{gathered}
$$

Можно ввести понятие величин эффективности влияния арматуры по каждому направлению, которые равны 
Таблица 1. Значения параметров по результатам моделирования

\begin{tabular}{c|c|c|c|c|c|c|c|c}
\hline \multirow{2}{*}{$\mu_{1}$} & \multicolumn{3}{|c|}{ Структура $1(k=8 \%)$} & \multicolumn{3}{|c}{ Структура $2(k=4 \%)$} \\
\cline { 2 - 9 } & $\mu_{z}, \mu_{y}$ & $\mu_{x}$ & $\eta_{z}, \eta_{y}$ & $\eta_{x}$ & $\mu_{z}, \mu_{y}$ & $\mu_{x}$ & $\eta_{z}, \eta_{y}$ & $\eta_{x}$ \\
\hline 1.1 & 1.008 & 1.008 & 0.98 & 0.96 & 1.004 & 1.004 & 0.98 & 0.96 \\
2 & 1.067 & 1.056 & 0.84 & 0.70 & 1.033 & 1.028 & 0.83 & 0.70 \\
3 & 1.12 & 1.087 & 0.76 & 0.54 & 1.060 & 1.043 & 0.75 & 0.54 \\
5 & 1.22 & 1.12 & 0.68 & 0.38 & 1.11 & 1.060 & 0.67 & 0.37 \\
10 & 1.43 & 1.16 & 0.60 & 0.21 & 1.21 & 1.077 & 0.60 & 0.21 \\
100 & 5.05 & 1.20 & 0.51 & 0.031 & 3.02 & 1.097 & 0.51 & 0.025 \\
1000 & 41.05 & 1.20 & 0.50 & 0.003 & 21.02 & 1.10 & 0.50 & 0.003
\end{tabular}

отношениям эффективных коэффициентов заполнения по каждому из направлений к фактическому коэффициенту заполнения: $\eta_{z}=k_{z} / k, \eta_{y}=k_{y} / k, \eta_{x}=k x / k$.

Предельный случай: если $\mu_{1} \rightarrow \infty$, то $k_{z} \rightarrow k_{x \mathrm{~A}} k_{y \mathrm{~A}}$, $k_{y} \rightarrow k_{x \mathrm{~B}} k_{z \mathrm{~B}}, k_{x} \rightarrow 0$. Если при этом $k_{y \mathrm{~A}}=k_{z \mathrm{~B}}$ и $k_{x \mathrm{~A}}=k_{x \mathrm{~B}}$ (типичный частный случай одинаковой укладки стержней в обоих направлениях), то $k_{x \mathrm{~A}} k_{y \mathrm{~A}}=k_{x \mathrm{~B}} k_{z \mathrm{~B}}$, и $\eta_{z}=\eta_{y} \rightarrow 0.5$, т. е. эффективные коэффициенты заполнения в плоскости укладки в пределе равны половине фактического коэффициента заполнения. Эти значения соответствуют принятым ранее в подходе для расчета поля в модели ММТС1 коэффициентам $k_{z}=k_{y}=0.5 k$.

Рассмотрим типичные решетчатые структуры с коэффициентами $k$ заполнения сталью $8 \%$ и 4\%. Структура 1: $k=8 \%, \Delta x=\Delta y=\Delta z=10 \mathrm{~cm}, \Delta x_{\mathrm{A}}=\Delta x_{\mathrm{B}}=$ $=\Delta y_{\mathrm{A} 1}=\Delta z_{\mathrm{B} 1}=2 \mathrm{~cm}$. Структура $2: k=4 \%, \Delta y=\Delta z=$ $=25 \mathrm{~cm}, \Delta x=8 \mathrm{~cm}, \Delta x_{\mathrm{A}}=\Delta x_{\mathrm{B}}=\Delta y_{\mathrm{A} 1}=\Delta z_{\mathrm{B} 1}=2 \mathrm{~cm}$.

Были проведены численные расчеты трехмерного распределения магнитостатического поля в ячейках периодичности обеих решетчатых структур. С учетом симметрии в качестве расчетных областей выбраны области, в восемь раз меньшие ячеек периодичности, $5 \times 5 \times 5 \mathrm{~cm}$ и $4 \times 12.5 \times 12.5 \mathrm{~cm}$ соответственно. Эти области включали соответственно $1 \cdot 10^{6}$ и $6 \cdot 10^{6}$ конечных элементов. Границы расчетных областей лежат в плоскостях, проходящих через центральные оси стержней. Моделирование проводилось независимо для двух вариантов наперед заданной (с помощью граничных условий) ориентации и величины среднего поля: параллельно одному из стержней (оси Z) и перпендикулярно обоим стержням (параллельно оси $X$ ). Напряженность среднего поля была принята 10 Ое. Значения магнитной проницаемости стержней задавались в диапазоне от 1.1 до 1000. По результатам моделирования определялась средняя индукция $\left(B_{z}\right.$ или $B_{x}$ соответственно) как отношение магнитного потока через соответствующее сечение расчетной области к площади этого сечения. Эффективная магнитная проницаемость $\left(\mu_{z}\right.$ или $\left.\mu_{x}\right)$ ячейки периодичности, а следовательно, и эквивалентной однородной среды, определялась как отношение вычисленной средней индукции к заданной средней напряженности поля. Результаты представлены в табл. 1.

Можно видеть из таблицы, что в предельном случае $\mu \rightarrow \infty$ эффективности влияния арматуры совпадают с полученными выше аналитическим путем значениями: $\eta_{z} \rightarrow 0.5, \eta_{y} \rightarrow 0.5, \eta_{x} \rightarrow 0$ (т. е. $\mu_{z}, \mu_{y} \rightarrow 0.5 k \mu, \mu_{x} \rightarrow 1$ ).

В общем случае наиболее целесообразным способом определения эффективных свойств арматуры представляется численное моделирование поля в ячейке периодичности. Это позволяет без каких-либо упрощающих предположений учесть дополнительно особенности арматуры, например, форму поперечного сечения стержня, наличие или отсутствие контакта между стержнями и т. п.

\section{Модифицированная изотропная модель решетчатой структуры}

В практических расчетах использование нелинейных анизотропных свойств материалов может представлять определенные сложности. Однако анизотропные свойства решетчатой структуры можно сымитировать, применяя модифицированную специальным образом изотропную модель.

Пусть $\mu_{\mathrm{A} 1}=\mu_{\mathrm{B} 1}=\mu_{1}$ и $k_{y \mathrm{~A}}=k_{z} \mathrm{~B}, k_{x \mathrm{~A}}=k_{x \mathrm{~B}}$. В этом случае анизотропная среда описывается эффективными коэффициентами заполнения по каждому из трех направлений, в которых $k_{z}=k_{y}$ в силу $\mu_{z}=\mu_{y}=\mu^{*}$. Такую среду можно заменить эквивалентной слоистой структурой, состоящей из двух чередующихся однородных изотропных слоев, параллельных плоскости $Y Z$, один из которых является магнитным, а другой - немагнитным.

Задача заключается в нахождении такого коэффициента заполнения магнитного слоя сталью $k_{1}$ и коэффициента заполнения среды магнитными слоями $k_{2}$, чтобы эффективные значения $\mu_{x}$, и $\mu^{*}$ двуслойной структуры совпадали с анизотропными характеристиками решетчатой структуры. Исходя из требований непрерывности компонент векторов индукции и напряженности на границах разделов сред и баланса потоков магнитного поля,

Таблица 2. Значения параметров изотропной модели по результатам численного моделирования

\begin{tabular}{c|c|c|c|c|c|c}
\hline \multirow{2}{*}{$\mu_{1}$} & \multicolumn{2}{|c|}{ Структура $1(k=8 \%)$} & \multicolumn{3}{c}{ Структура 2 $(k=4 \%)$} \\
\cline { 2 - 7 } & $\mu_{k 1}$ & $k_{1}$ & $k_{2}$ & $\mu_{k 1}$ & $k_{1}$ & $k_{2}$ \\
\hline 1.1 & 1.03 & 0.286 & 0.273 & 1.023 & 0.227 & 0.1726 \\
2 & 1.27 & 0.267 & 0.252 & 1.226 & 0.226 & 0.1478 \\
3 & 1.52 & 0.260 & 0.234 & 1.452 & 0.2260 & 0.1332 \\
5 & 2.01 & 0.253 & 0.214 & 1.898 & 0.2244 & 0.1196 \\
10 & 3.22 & 0.246 & 0.195 & 3.005 & 0.2227 & 0.1069 \\
100 & 24.6 & 0.239 & 0.171 & 22.82 & 0.2204 & 0.0927 \\
1000 & 23.9 & 0.238 & 0.168 & 220.9 & 0.2201 & 0.0911
\end{tabular}



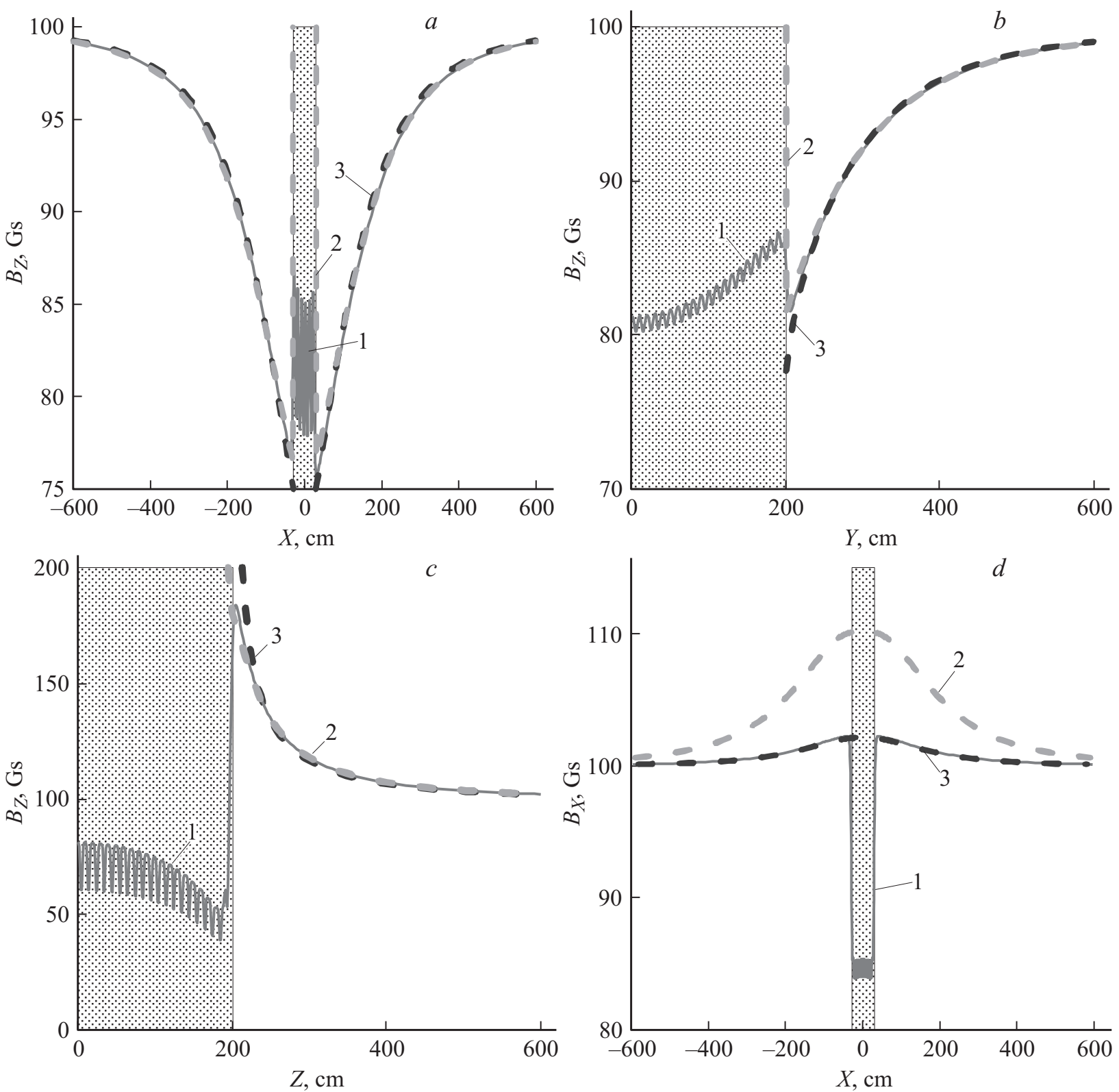

Рис. 3. Поле вдоль оси $X(a, d), Y(b, e), Z(c)$ при внешнем поле $100 \mathrm{G}$, параллельном оси $Z(a-c)$ и оси $X(d, e)$. Модели: $1-$ детальная, 2 - изотропная, 3 - модифицированная изотропная.

можно получить:

$$
\begin{gathered}
\mu^{*}=1+k_{2}\left(\mu_{k 1}-1\right), \\
1 / \mu_{x}=1-k_{2}\left(1-1 / \mu_{k 1}\right),
\end{gathered}
$$

где $\mu_{k 1}$ - эффективная проницаемость магнитного слоя.

Из (9) и (10) можно получить

$$
\begin{aligned}
\mu_{k 1} & =\left(\mu^{*}-1\right) /\left(1-1 / \mu_{x}\right), \\
k_{2} & =\left(\mu^{*}-1\right) /\left(\mu_{k 1}-1\right) .
\end{aligned}
$$

Эффективная магнитная проницаемость $\mu_{k 1}$ аналогично (4) связана с коэффициентом заполнения магнитного слоя сталью $k_{1}$ соотношением

$$
\mu_{k 1}=1+k_{1}\left(\mu_{1}-1\right),
$$

и, следовательно:

$$
k_{1}=\left(\mu_{k 1}-1\right) /\left(\mu_{1}-1\right) .
$$

Зная значение $\mu_{1}$ и геометрические параметры решетки, можно найти $\mu_{x}, \mu_{y}$ и $\mu_{z}$, и далее, по формулам $(11)-(14)-$ значение $\mu_{k 1}$ и параметры слоистой структуры $k_{1}$ и $k_{2}$.

Если конструкция имеет относительно малую толщину $\Delta x_{w}$, ее можно описать одним однородным магнитным слоем толщиной $k_{2} \Delta x_{w}$ и коэффициентом заполнения сталью $k_{1}$. Если толщина конструкции не может считаться малой, она описывается набором слоев суммарной толщиной $k_{2} \Delta x_{w}$ и коэффициентом заполнения $k_{1}$ в каждом слое. По формулам (11)-(14) были 


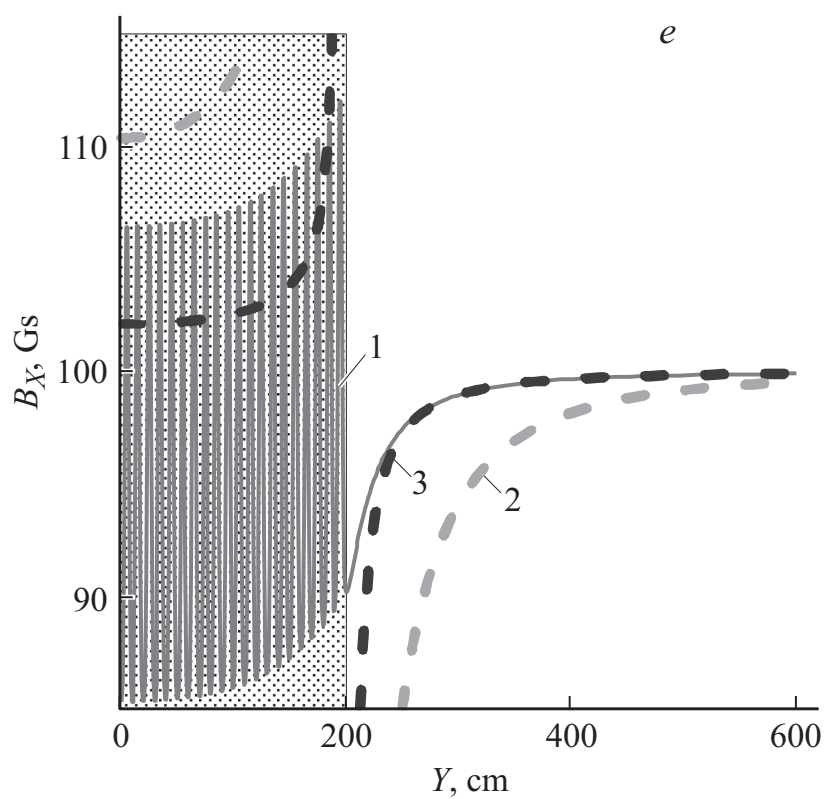

Рис. 3 (продолжение).

вычислены параметры слоистых структур $\mu_{k 1}, k_{1}, k_{2}$, для типичных решетчатых структур (табл. 2).

Из табл. 2 следует, что параметры слоистой структуры зависят от проницаемости стержней, которая в общем случае может быть функцией среднего поля в ячейке. Однако, как видно, коэффициенты $k_{1}$ и $k_{2}$ имеют пологую зависимость от $\mu_{1}$. Например, для структуры 1 в области наиболее типичных значений $\left(\mu_{1}>100\right)$ вариации $k_{1}$ составляют около $0.5 \%$ : $0.239 \pm 0.001$, а $k_{2}$ - около 1\%: $0.170 \pm 0.002$. Это позволяет для многих случаев построить модель решетки стержней с проницаемостью $\mu_{1}=\mu_{1}(H)$ в виде слоистой структуры с коэффициентами заполнения $k_{1}$ и $k_{2}$, которые соответствуют типичным значениям $\mu_{1}$.

\section{Решение модельных задач}

Для верификации предложенного подхода проведены расчеты модельных задач о возмущении однородного внешнего магнитного поля плитой, армированной стальными стержнями. Параметры решеток соответствовали структурам 1 и 2 (табл. 2). В примере 1 (со структурой $1, k=8 \%$ ) размеры плиты составляли $0.6 \times 4 \times 4 \mathrm{~m}$. В примере 2 (со структурой $2, k=4 \%$ ) размеры плиты $-0.48 \times 4 \times 4 \mathrm{~m}$. Относительная магнитная проницаемость стержней арматуры в обоих примерах выбрана постоянной, равной $\mu_{1}=100$. Центр системы координат расчетной модели совмещен с геометрическим центром плиты. Ее протяженные стороны направлены вдоль осей $Z$ и $Y$. Стержни в плите уложены параллельно осям $Z$ и $Y$.

Рассматривались два случая ориентации внешнего поля относительно плиты: параллельного и перпендикулярного плоскости укладки стержней. Величина внеш- него однородного поля была выбрана $100 \mathrm{G}$. Исходя из симметрии системы при выбранных ориентациях внешнего поля, расчетная область ограничена $1 / 4$ системы $(Y \geq 0, Z \geq 0)$. Решение каждой задачи получено с использованием детальной, изотропной и модифицированной изотропной моделей.

Детальные модели для примеров 1 и 2 содержат $2.5 \cdot 10^{7}$ и $2.8 \cdot 10^{7}$ конечных элементов соответственно. Для изотропных моделей размерность задачи существенно меньше $-3.7 \cdot 10^{6}$ и $3.5 \cdot 10^{6}$ элементов. Для модифицированной изотропной модели параметры эквивалентного слоя выбирались из табл. 2 и соответствовали $\mu_{1}=100$. Для примера 1 коэффициент заполнения слоя $k_{1}=0.24$, коэффициент заполнения слоями $k_{2}=0.17$, что соответствует суммарной толщине эквивалентного слоя $60 \mathrm{~cm} \times 0.17=10.2 \mathrm{~cm}^{2}$. Для примера $2 k_{1}=0.22$ и $k_{2}=0.093$, а суммарная толщина эквивалентного слоя $-48 \mathrm{~cm} \times 0.093=4.464 \mathrm{~cm}^{2}$.

Результаты моделирования демонстрируют сложный характер распределения поля внутри плиты, обусловленный влиянием отдельных стержней. Однако уже на расстояниях от плиты, сравнимых с шагом укладки стержней, характер распределения поля становится гладким, что позволяет отказаться от детального описания стержней в глобальных моделях. На рис. 3, $(a-c)$ видно, что при внешнем поле, параллельном одному из направлений укладки стержней (вдоль оси $Z$ ), все три модели на расстояниях, больших нескольких шагов периодичности, дают практически одинаковый результат. В случае внешнего поля, перпендикулярного к плоскости укладки стержней, для изотропной модели имеет место завышение уровня собственного поля арматуры в 5-7 раз. В зависимости от направления собственного поля, это приводит либо к завышению (рис. $3, d$ ), либо к занижению (рис. $3, e$ ) результирующего поля на $8-10 \%$. В отличие от изотропной модели, модифицированная модель демонстрирует существенно более точный учет влияния арматуры. Погрешность в оценке собственного поля арматуры составляет не более $1 \%$ на расстояниях, сопоставимых с шагом арматуры. Погрешность определения суммарного поля при этом составляет не более $0.1 \%$.

\section{Заключение}

Предложена методика расчета поля армированных сталью бетонных конструкций, обладающих анизотропией магнитных свойств, обусловленной особенностями укладки стальных стержней арматуры.

Для ячейки периодичности арматуры в виде прямоугольного параллелепипеда получены упрощенные аналитические выражения, связывающие эффективные (усредненные) значения индукции, напряженности и магнитной проницаемости.

Разработан алгоритм и выполнены численные расчеты пространственного распределения поля в ячейке пе- 
риодичности, которые сопоставлены с аналитическими выражениями. Показано, что численное моделирование является общим и эффективным способом определения усредненных магнитных свойств армированных конструкций. Предложен подход, который позволяет свести анизотропную модель для описания армированных конструкций к слоистой структуре, состоящей из чередующихся магнитных и немагнитных однородных изотропных слоев, что упрощает проведение расчетов.

Выполнен анализ результатов решения важной для практики модельной задачи о возмущении однородного внешнего магнитного поля плитой, армированной стальными стержнями. Он показал, что модифицированная изотропная модель (использующая слоистые структуры) обеспечивает требуемую при создании токамаков точность при любой ориентации внешнего поля. Для модифицированной и изотропной моделей размерности задач и вычислительные затраты сопоставимы.

\section{Список литературы}

[1] International Thermonuclear Experimental Reactor www.iter.org

[2] Amoskov V., Belov A., Belyakov V., Gribov Yu., Kukhtin V., Lamzin E., Maximenkova N., Sytchevsky S. // Plasma Devices Operations. 2008. Vol. 16. N 4. P. 225-233.

[3] Amoskov V., Belov A., Belyakov V., Gribov Yu., Kavin A., Kukhtin V., Lamzin E., Lobanov K., Maximenkova $N$., Mineev A., Sytchevsky S. // Plasma Devices and Operations. 2009. Vol. 17. N 4. P. 238-249.

[4] Amoskov V., Belov A., Belyakov V., Gribov Yu., Kukhtin V., Lamzin E., Maximenkova N., Sytchevsky S. // Plasma Devices Operations. 2009. Vol. 17. N 4. P. 230-237.

[5] Belov A.V., Belyakova T.F., Gornikel I.V., Kukhtin V.P., Kuchinsky V.G., Lamzin E.A., Semchenkov A.G., Shatil N.A., Sytchevsky S.E. // IEEE Transactions on Applied Superconductivity. 2008. Vol. 18. N 2. P. 1609-1612.

[6] Amoskov V.M., Belov A.V., Belyakov V.A., Belyakova T.F., Gribov Yu.V., Kukhtin V.P., Lamzin E.A., Sytchevsky S.E. // Plasma Devices Operations. 2008. Vol. 16. N 2. P. 89-103.

[7] Amoskov V., Belyakov V., Bazarov A., Kaparkova M., Kukhtin V., Lamzin E., Lyublin B., Sytchevsky S. Modeling magnetic effects of steel rebar of concrete surroundings for electrophysical apparatus Proc. RuPAC 2016. St. Petersburg, Russia, P. 553-555. 